\title{
ARQUITECTURA INCA A TRAVES DE SUS TEXTILES PERU- FAUA/UNI
}

\author{
INCA ARCHITECTURE THROUGH YOUR TEXTILES \\ PERU- FAUA / UNI
}

\author{
Walter Gonzales Arnao ${ }^{1}$
}

RESUMEN

\begin{abstract}
Proponemos una mirada a los textiles incas como soporte planímetro de su territorio e inducir a reflexionar sobre las probables aplicaciones de los textiles incas en arquitectura y poner en evidencia a los ojos de los diseñadores, arquitectos e ingenieros, la hipótesis del valor y aplicación de los textiles incas en la representación planimetría de la tridimensionalidad del mundo material como lo concebian. Ensayar ideas sobre los usos del arte de los telares y su influencia en la arquitectura Inca. Utilizar este conocimiento milenario es un instrumento de inspiración de los futuros arquitectos.
\end{abstract}

Palabras clave.- Arte textil inca, Arquitectura, Revalorar tecnología textil originaria, Reflexión estética textil, Aplicación actual como inspiración.

\begin{abstract}
We propose a look at incas textiles and planimetric support of its territory an Inducing reflection on the possible applications of textiles in architecture incas, and bring out the eyes of designers, architects and engineers with the hypothesis of the value and application of incas textiles in representing three-dimensional surveying of the material world as conceived. Test ideas about the uses of the art of weaving and its influence on Inca architecture. Use this ancient knowledge an instrument of inspiration for future architects.
\end{abstract}

Key words.- Inca textile art, Architecture, Textile technology reassessment, Aesthetic reflection textile, Current application as inspiration.

\section{INTRODUCCION}

La producción textil en la cultura inca tuvo una relevante aplicación, según las crónicas españolas y las ilustraciones de Felipe Guamán Poma de Ayala, donde hay un consenso según las investigaciones de los antropólogos, en la función que cumplían de simbolizar estatus, estirpe, región de procedencia e información no decodificada de los tocapus, también eran usados como preciados objetos de intercambio, así mismo se reconoce el alto valor que le daban en ceremonias y negociaciones diplomáticas entre las naciones para cerrar acuerdos. Según las crónicas se afirma que a la muerte de cada inca se negociaba nuevamente y se renovaban los acuerdos y se sellaban con intercambio de mantos. Los estudios del Arq. Carlos Milla V. sobre la iconografía sagradas andina, se puede notar como síntesis de la utopía y cosmovisión reflejados en los textiles, cerámicos y arquitectura. Asimismo, el Arq. C. Enrique Guzmán García, deslizó el concepto donde "trama y el urdiembre de los telares textiles", corresponden al trazado del emplazamiento de los andenes incas en el Valle Sagrado del Huatanay al oeste del Cusco (TICAPATA - LARAPA Y PATA PATA), donde literalmente identifica una intensión adrede y hace notar que son el resultado de todo un diseño previo. Así mismo el Arq. Adine Gavazi, menciona que el paisaje como texto y tejido como binomio, es inseparable en la cultura

\footnotetext{
${ }^{1}$ Arq. Docente investigador de la Facultad de Arquitectura, Urbanismo y Artes de la Universidad Nacional de Ingeniería.
} 
andina y amazónica del Perú [1].

La dimensión que realmente tuvieron los textiles, incluidos los quipos incas, no han sido decodificados totalmente, a todas luces las claves para comprenderlos se perdieron en la conquista. En este sentido la ingeniería tal como la conocemos, a través de las matemáticas tiene mucha relación con las matrices generadas de los textiles y sus correlaciones iconográficas recreadas en los tocapus.

También desde el punto de vista del diseño como lo conocemos en la elección del color para la urdiembre, el tipo de hilo empleado, así como el punto de la textura y finalmente la geometría compleja empleada, implica un alto nivel de abstracción en composición, si lo analizamos desde el punto de vista de la estética, como si fuera un lienzo de caballete, esta interpretación no es la aproximación correcta.

Esta aproximación a la arquitectura a través de los telares, nos plantea interrogantes muy importantes para la ingeniería y arquitectura, como si efectivamente los mantos fueron usados como representación planimetría de la tridimensionalidad, reflejado en arquitectura.

Los puentes colgantes tejidos con fibras vegetales le merecen especial atención a la arquitectura y la ingeniería donde usaron la tecnología de los telares, para resolver unos de los sistemas viales más eficientes del mundo antiguo, según el Dr. John A. Ochsendorf, del Instituto Tecnológico de Massachusetts especialista en arquitectura e ingeniería antigua, tales puentes cubrían más de 50 a 80 metros, de luz en quebradas verticales, solo en china y el Himalaya se construyeron algo parecido en el siglo 3 a.d.c, con cadenas de hierro en luces menores.

El diseño de los tejidos en telares y la relación de lo unidimensional del hilo al bidimensional de los textiles y la tridimensionalidad de los puentes colgantes donde el denominador común es el trabajo de la tensión de la fibra natural, nos da un rumbo para comprender estas construcciones, sacando lecciones de ingenio y creatividad de estas obras y nos invita a establecer nexos aplicativos para problemas actuales y su relación con la arquitectura.

\section{PLANIMETRÍA Y TRIDIMENSIONALIDAD DE LOS TELARES INCAS}

Los planos y mapas de ingeniería y arquitectura que conocemos en la actualidad manejan convencionalismos que reconocemos, aceptamos y tienen consenso general, estas claves son los simbolismos que representamos en los planos para identificar las puertas, muros, niveles, caminos, etc. De la misma manera que para entender planos mapas de arquitectura y ingeniería se requiere de una formación técnica especializada. En las grandes civilizaciones e imperios de todas las épocas de la historia el manejo de la información de lectura de mapas, y levantamiento topográfico de emplazamientos humanos territoriales estuvo ligado a una rigurosidad y un uso militar por la importancia que significa conocer con precisión el territorio conquistado para poder controlarlo. Aplicando este criterio los incas tuvieron necesariamente que tener una representación planimetría de los territorios conquistados, es decir una suerte de planos o mapas que representaran sus dominios, caminos, etc. Los textiles son el soporte pertinente dado que los incas dominaron técnicas avanzadas, para poder representar geometrías complejas, abstractas y que muy bien podría representar lo más parecido a un plano o mapa, con claves o simbolismo que no se han descifrado aun. Adine Gavazzi. afirma "que los tejidos comunican un paisaje en miniatura y son realizados como mapas geométricos y geográficos que expresan un modo de representar un espacio similar a un texto y un código visual.Ya sea como texto que como tejido el paisaje es reconocido y mapeado componiendo muchos elementos" para este efecto pone como ejemplo "las salinas de Maras en el Valle de Cuzco donde juntan terrazas entretejida a altura diferentes en el paisaje (Fig.1), y la composición de las parcelas de cultivo siguen un criterio biomorfico reticular, que está presente desde épocas arcaicas en los textiles (Fig2)", en resumidas cuentas la relación con el paisaje fue tratado como la aplicación de un urdiembre y una trama que forma un tejido en el territorio. A su vez el Arq. C. Enrique Guzmán, dice respecto a esta interpretación que - "los ejemplos a los que se refiere la arquitecta Gavazzi, son consecuencia de una superposición y adición natural de unas terrazas con otras y que a los ojos actuales de la arquitecta, hace esa comparación metafórica". 
Mientras que los ejemplos que el Arq. C. Enrique Guzmán, que menciona en su tesis, si se tratan de extensiones de andenes y terrazas diseñados deliberadamente, dice: "Ticapata-Larapa y Pata Pata”, parte de la expansión este del Cuzco, presenta terrazas que asemejan tejidos tanto llanos en cuadricula como escalonamientos intencionales tipo chacana....en el arte inca predomina el diseño geométrico, como se ve en la cerámica el arte textil y el labrado y tallado de las piedras", la representación planimétrica literal del textil en arquitectura es confirmada en esta tesis [2]. La arquitectura y territorio pudieron haberse representado no solo en textiles, sino en otro medio (Guzmán 1998).

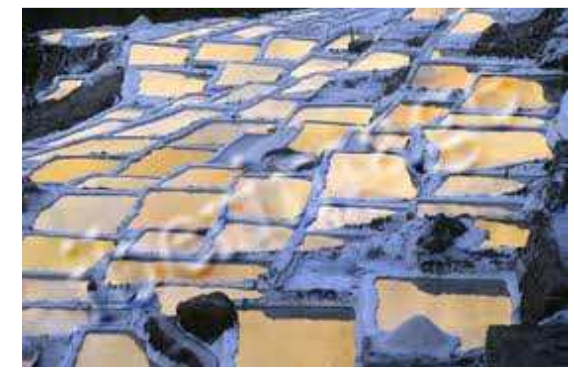

Fig. 1 Salares incas.

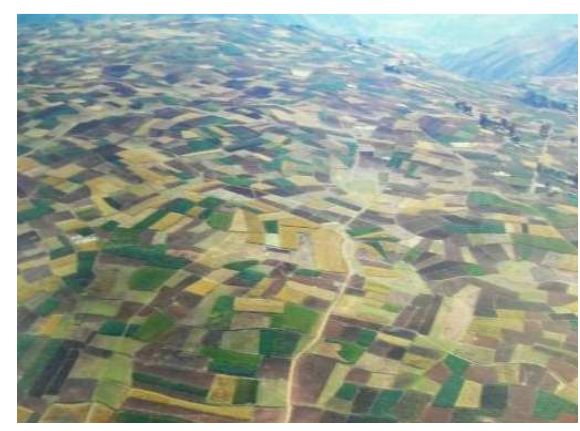

Fig. 2 Disposición territorial andina bajo el concepto de trama y urdiembre.

Los textiles o mantos incas como se puede apreciar tienen un trabajo que va más allá de los límites utilitarios de cubrirse del frio, y son un lienzo lleno de simbolismo sagrado y pragmático que contiene información geográfica de espacialidad y pertenencia regional, tal como postula Alejandro Vega Ossorio "que los símbolos de las vestimentas de las elites indicaban cada región que conquistaban o controlaban, asimismo agrega que se han identificado 294 diseños de tocapus que básicamente son pequeños cuadrados, distribuidos cual tablero de ajedrez, las Acllas tejían prendas para la elite con tocapus", con un alto nivel de abstracción simbólico, que dominaban. Según las crónicas el inca Wiracocha introduce la diferencia de vestimenta para diferenciar la región y estatus de procedencia esto incluye colores y iconografía, el uncu inca (Fig. 3) con los tocapus sufren modificaciones a la llegada de los españoles como se aprecia en el unco (Fig. 4 y Fig. 5), en esta últimas dos figuras, se observa imágenes que recrean aparentemente plantas y caminos, esto se podría explicar porque el extirpador de idolatrías prohibió el uso de iconografía inca que, los llamados uncos modificaron su simbología haciéndolas más figurativas.

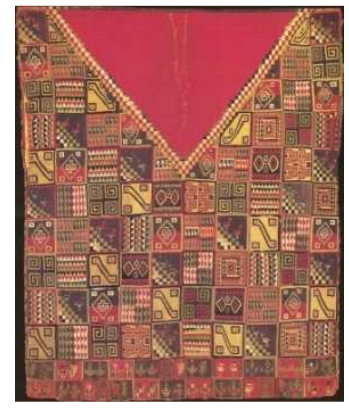

Fig. 3 Unco inca-poncho.

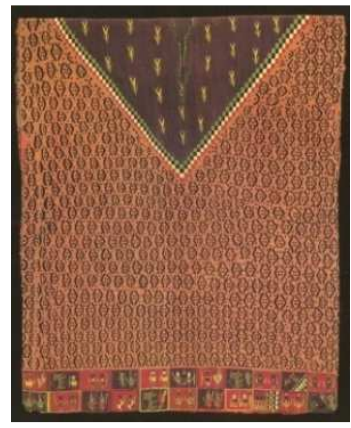

Fig. 4 Unco virreynal-poncho.

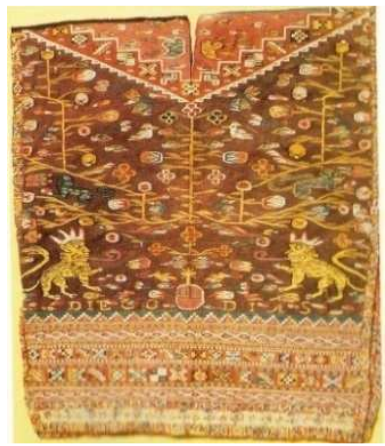

Fig. 5 Unco republicano-poncho. 


\section{ARTE TEXTIL Y ARQUITECTURA- INGENIERIA-ESPACIALIDAD}

"How the Inca Leapt Canyons" es el título del articulo del THE NEW YORK TIMES publicado el 08 de mayo 2007 por John Noble Wilford, que traducido al pie de la letra significa "Cómo el Inca Saltaba Cañones”, donde se da a conocer la experiencia de estudiantes del Massachusetts Institute of Technology, en la construcción de un puente colgante inca con el objeto de conocer la tecnología desarrollada, a base de un material (ichu o paja) que ha sido mantenido hasta nuestros días por la renovación anual de los pobladores Queswachaca - Cuzco. Mediante sus estudios del Dr. Ochsendorf, se ha podido analizar que "La Cultura Inca, fue la única antigua civilización americana que llegó a fabricar puentes colgantes". Algunos puentes similares fueron también construidos en el siglo 3 a.d.c. en otras zonas montañosas del mundo, especialmente en el Himalaya y la China, pero fueron fabricados con cadenas de hierro. Y agrega que esta tecnología del empleo de fibras vegetales es única en su tipo, como una aplicación para cubrir luces grandes no era conocida en Europa. Esto explica el asombro intimidante que según las crónicas tenían los españoles al pasar por estos puentes colgantes que con el viento se agitaban como una hamaca gigante, sobre laderas verticales. En la imagen de la Fig. 6, se aprecia que la aves vuelan por debajo del puente dando una dimensión, de la profundidad del cañón.

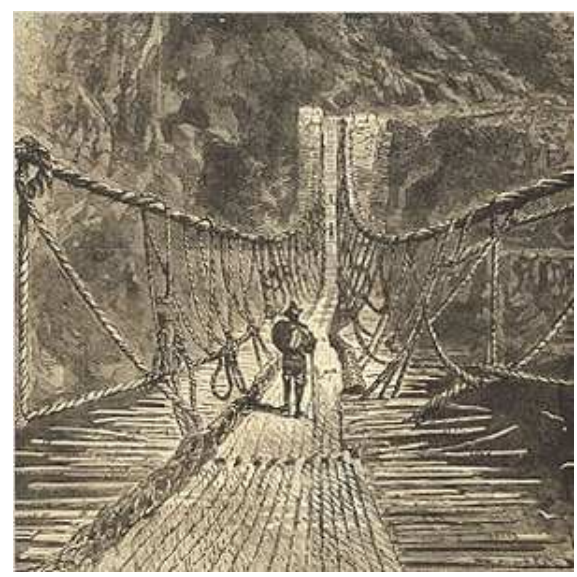

Fig. 6 E. George Squier (Harper and Brothers) Grabado, de "Perú: Incidentes de viaje y exploración en la Tierra de los Incas" Un puente colgante Inca en 1877 [3].
El análisis de la arquitectura inca se basa en el estudio de la piedra en compresión, poco se ha escrito sobre tracción de los puentes colgantes y su relación con la arquitectura e ingeniería salvo su utilidad en el Capac Ñan.

Esta dualidad (compresión y tracción) de lo pétreo y ligero que se da en lo temporal de lo duradero de la piedra y la corta duración de esta fibra de estos puentes, nos invita a reflexionar sobre el papel del manejo de técnicas de izamientos en altura de pesadas mampostería, en geografía verticales. Es decir, el manejo y aplicación de la tridimensionalidad de la fibra y la versatilidad de su uso llevando al límite estructural en aplicaciones de ingeniería. Este manejo espacial de la fibra, se da también en el uso y lectura de los quipus, que definitivamente se leía tridimensionalmente, que era parte de cómo entendían el mundo [4]. Otro ejemplo emblemático es el trabajo en los mates burilados, que son superficies esféricas entorno al cual se desarrolla toda una suerte de comic donde cada imagen guarda relación con la anterior contando una historia que finaliza con una imagen tallada en la tapa, esta temporalidad espacial, mesclada con el mundo mágico de su cosmovisión, nos dice una manera de entender el espacio y tiempo, que se refleja en esta producción artística. El lienzo del pintor de caballete europeo no tiene esta espacialidad tridimensional porque trabaja en un plano, usando la perspectiva como recurso de manejar la tridimensionalidad. En el caso de las vestimentas textiles de los llamados Uncos, si bien es cierto su producción, era en telares planos, su diseño estaba pensado para vestimentas que una vez en el cuerpo del usuario tomaba un carácter tridimensional que era la manera cotidiana de ver estos trajes, de toda la población inca, esto nos induce a pensar que así como para entender un mate burilado tienes que rodearlo para conocer toda la historia, de la misma manera para conocer realmente al portador de la vestimenta tenías que rodearlo para reconocer toda la simbología que tenían estos Uncu, que como ya se preciso tiene significado.

Entonces los textiles y lo tejidos del puente colgante encierran la conexión de cómo entendían y hacían arquitectura, porque visualizaban la tridimensionalidad en una forma diferente a la que nosotros la entendemos. Entonces los arquitectos 
incas necesariamente tuvieron que recurrir a los textiles como una herramienta más para trabajar la arquitectura como la de Machupichu, que hasta hoy en día para levantar los planos de este santuario es complejo en una arquitectura que se confunde con la naturaleza y más aún si te encargan diseñar en pendientes más fuertes, como lo hicieron ellos.

\section{EXPERIENCIA CON JOVENES DE LA UNI CON EL ARTE TEXTIL (TELARES)}

La iniciación de los alumnos en el dominio de estas técnicas, para luego expresar su sensibilidad estética, es compleja por las diferentes motivaciones que atrae el llevar el curso, pasando por los que realmente están interesados en dominar este arte, hasta los que lo llevan para completar créditos. (Ver Fig. 7).

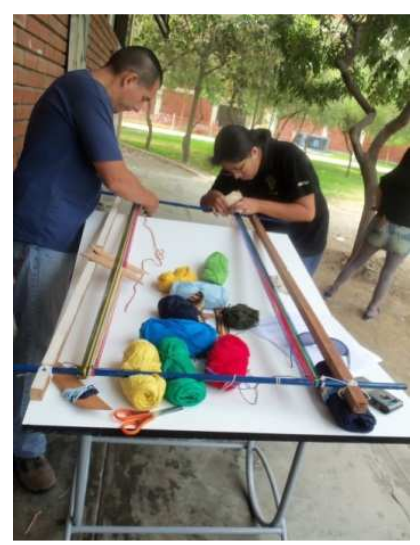

Fig. 7 Ejercicios con telar de cintura.

También se enfrentan al pensamiento que los telares o los tejidos es para mujeres, cuando es sabido que en los andes los hombres son los que ejercen esta práctica (ejemplo Taquile, selva peruana, etc.) (Ver fig. 8).

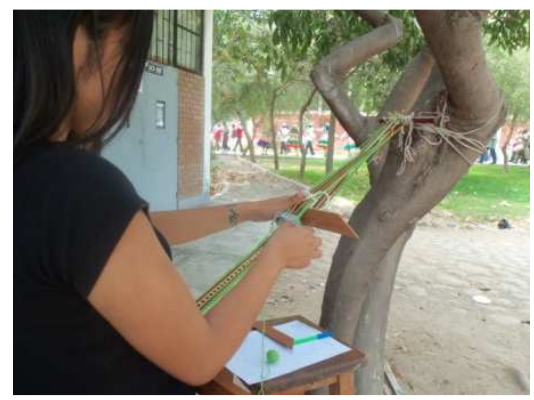

Fig. 8 Combinación de colores con las tecnicas del telar de cintura.
A este respecto puedo contar que un alumno del curso de telares fue objeto de burla por parte de su padre al verlo tejer en telar de cintura. Obligando a este alumno a realizar la práctica del telar a escondidas de sus padres y amigos. Este tipo de pensamiento que conduce a la poca valoración de esta producción artística se debe al nivel que ocupa en nuestra sociedad los llamados artesanos, que son practicadas por campesinos en los andes para su uso o para el turismo.

Las matrices con sistemas binarios que se emplea para geometrizar los diseños, despierta el interés en los alumnos de la FAUA de la preparación matemática, donde se pone a prueba la complejidad de la técnica y los limites de diseño que pone el alumno, siendo una ventana creativa con mucho potencial en composición, donde se diseña desde el momento de seleccionar los colores de los hilos, texturas geometrías, concepto de diseño, etc. Esta versatilidad que tiene el telar peruano que hace en los jóvenes universitarios FAUA, despierta un interés especial.

Otro de los aspectos que causó particular atención en los alumnos es su memoria colectiva en un sector de descendientes de provincianos que tenían familiares o conocían que habían trabajado en telares con técnicas incas o mestizas y que considero que esta es otra de las causas por la que el alumno se siente atraído por el curso.

\section{TELAR PORTATIL Y FAB LAB, PARA LA ENSEÑANZA DE ARTES APLICADAS PERUANAS}

El evento realizado en la UNÍ en la Facultad de Arquitectura Urbanismos y Artes, bajo la dirección del decano Luis Delgado Galimberti, el denominado ENCUENTRO INTERNACIONAL FAB 7 LIMA, convocó a expertos en diseño digital, abrió un espacio para desarrollar un modelo de telar portátil (ver Fig. 9), que es una manera de difundir (ver Fig. 10) nuestra cultura 5. Asimismo, se utilizó lo último en tecnología de diseño, es importante encontrar los caminos prácticos para sintetizas y simplificar nuestra legado cultural de toda la producción artística del Perú en todas las disciplinas, para difundir y peruanizar a los peruanos, algo como lo ocurrido con el arte gastronómico, que en la actualidad pasa por un buen momento que no tiene su reflejo en todas las artesanías de nuestro pueblo que vive un 
congelamiento cultural, que han sido confinadas a "guetos culturales o reservaciones aisladas" con los llamados CITES, o Centros De Innovación Tecnológica que lo único que hacen es congregar y dar apoyo técnico y no proyectan en el tema del diseño ni cultura en el entorno regional [5].

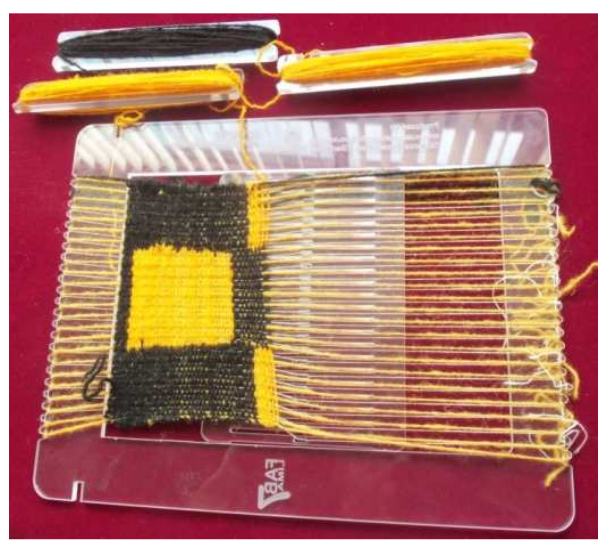

Fig. 9 Telar manual para la enseñanza del telar de cintura.

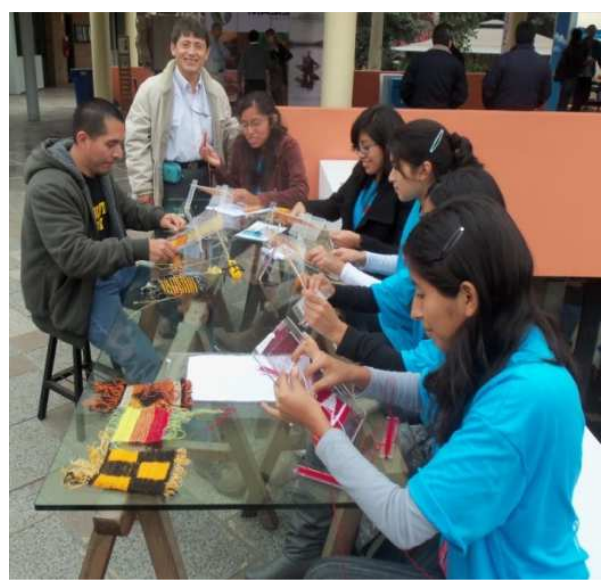

Fig. 10 Taller de telares con alumnos del segundo ciclo.

NOTA: La aplicación de la tecnología textil en la arquitectura no es novedad de los incas ya en Caral hace 5000 años lo usaron para construir edificios monumentales y garantizar estabilidad, con bolsas tipo maya de fibras vegetales llenas de piedras, llamadas shicras (como los gaviones en la actualidad). Según estas bolsas de piedra y los quipus encontrados en Caral, por Ruth Shady queda demostrado la larga tradición textil en la construcción y la cultura peruana que es la síntesis de más de 14 civilizaciones desarrolladas, que culminaron con los incas, donde se produce el quiebre cultural, con la conquista y que genero un congelamiento de estas artes y tradiciones constructivas.

\section{CONCLUCIONES}

El arte textil prehispánico cumplió la función de soporte planimétrico de la tridimensionalidad territorial y arquitectura, que en la actualidad también se manifiesta en los textiles de la cultura popular peruana.

Los tocapus de los uncos incas ponen en evidencia un claro dominio y un elevado nivel de abstracción que induce a pensar que pudieron ser un tipo de codificación o claves que representaba territorio y su cosmovisión mágico religioso en los textiles.

La tecnología de los telares, reflejados en los sistemas de isaje de mampostería de piedra en geografías verticales, así como en los puentes colgantes prehispánicos, fue importante en la arquitectura, civil, militar y religioso de la cultura inca.

Los arquitectos incas conocieron la tecnología textil y lo utilizaron como herramienta para facilitar su trabajo, en lo que hoy llamaríamos proceso de proyección del diseño, en una clara concepción diferente a la que trajeron los europeos.

El arte textil con técnicas ancestrales de producción artística, pueden servir como un medio de expresión y/o comprensión, estética de los jóvenes estudiantes de arquitectura, aplicando este conocimiento con una nueva visión de la arquitectura inca a través de los textiles.

El arte textil originario está latente en la cultura popular peruana que ha perdido su carácter mágico religioso de su cosmovisión, pero sigue latente en su sentido expresivo como producción artística.

La experiencia obtenida en la enseñanza del curso Telares en la Facultad de Arquitectura, de esta técnica textil ancestral, demostró el enorme potencial como medio de trasmisión de cultura peruana, utilizando todos los medios que nos ofrece la tecnología moderna.

Esta manifestación de producción artística en telares que en la actualidad sigue latente en todo el territorio peruano, que es conocido como arte popular, es un rezago de la importancia que 
siempre tuvo en el imaginario popular, que se puede utilizar como motivo de inspiración para los arquitectos del futuro, sin caer en un remedo o chauvinismo del movimiento indigenista con el llamado neo-peruano.

\section{REFERENCIAS}

1. Gavazzi, A., "Arquitectura Andina", Apus Grafh Ediciones, primera edición pp. 34-37

2. Ver http://www.raco.cat/index.php/BoletinAmeric anista/article/view/98733/146687

3. Ver http://www.larepublica.pe/11-06-2014/qeswachaca-el-ultimo-puente-inca-reconocidocomo-patrimonio-mundial

4. Gonzales Arnao, W., "Silabo Telares AFA 715”, Faua-Uni, 2011.

5. Ver

http://www.youtube.com/watch?hl=es\&v=OQ kopUco6S0

Correspondencia:

waltergonzalesarnao@gmail.com

Recepción de originales: setiembre 2014

Aceptación de originales: diciembre 2014 